\title{
Acquiring conjunctive concepts: When and why does feature frequency affect feature identification?
}

\author{
THOMAS C. TOPPINO \\ Villanova University, Villanova, Pennsylvania \\ and \\ NANCY M. BUCHER \\ University of California, Berkeley, California
}

\begin{abstract}
According to frequency theories of concept attainment, acquisition of rule-governed concepts depends on discriminating relevant from irrelevant stimulus features on the basis of their respective frequencies of occurrence. Depending on the theorist, the critical frequency counts may be based on occurrence in all stimuli or on occurrence in positive instances only. Experiment 1 held frequency in positive instances constant and varied the frequency of irrelevant features in the negative category. High frequency was associated with impaired performance, but only when concept problems started with a negative instance. Experiment 2 demonstrated that some previous findings in the literature that were thought to support frequency theory also depended on problems starting with a negative instance. These findings are not easily reconciled with existing frequency theories. We attribute our results to high intercategory similarity, which is a correlate of high frequency of irrelevant features. In the context of a hypothesis-testing theory, we propose that a negative start stimulus proactively interferes with memory for information in the initial positive instance and that the extent of the interference depends on how similar negative instances are to positive instances.
\end{abstract}

As Kellogg (1980) noted, strength or frequency theories have had a long history in the area of concept formation. Early theories were stimulusresponse theories, in which frequency of occurrence was a major determiner of associative strength (e.g., Hull, 1920; Restle, 1955). More recently, cognitive theorists have proposed that people store frequency information and use it as a basis for classification decisions. Such theories have been applied with some success to the formation and use of ill-defined concepts that have a prototypical structure. For example, stimuli have been shown to be recognized more confidently (Neumann, 1974), classified more accurately (Hayes-Roth \& Hayes-Roth, 1977), and rated as more typical of a category (Kellogg, Bourne, \& Ekstrand, 1978) to the extent that they contain features that occur more frequently in the category. In addition, several studies have supported the predictions of frequency theories over the predictions of alternative formulations (e.g., Chumbley, Sala, \& Bourne, 1978; Goldman \& Homa, 1977; Hayes-Roth

We would like to thank Mary Claps leni, Christine Wojciechowski, and Elvira Perewiznyk for their assistance in collecting data for Experiment 1. Requests for reprints should be sent to Thomas C. Toppino, Department of Psychology, Villanova University, Villanova, Pennsylvania 19085.
\& Hayes-Roth, 1977). Thus, although frequency theories are not the only current theories of the acquisition and use of ill-defined concepts, they are among the most prominent. In contrast, recent accounts of the formation and use of well-defined, rule-governed concepts have been dominated by hypothesis theories, and frequency theories have had relatively little influence.

According to hypothesis theories, people acquire concepts through a problem-solving process. They formulate hypotheses about which stimulus features may be relevant and then test their hypotheses against logical information that they receive subsequently. A well-defined concept is acquired or "solved" when the correct hypothesis is isolated. Acquisition or solution difficulty may depend on the rate at which logical information allows incorrect hypotheses to be eliminated and on the probability of testing the relevant stimulus features. However, hypothesis theories have not included the assumption that people store and use frequency-of-occurrence information in the formation of well-defined concepts (e.g., Bower \& Trabasso, 1964; Restle, 1962, Trabasso \& Bower, 1968). Because using frequency information seems to require a gradual learning process, emphasizing the importance of the frequency may have seemed to conflict with some of the assumptions un- 
derlying hypothesis theory. Moreover, a frequencybased process may have seemed difficult to reconcile with findings such as those of solution-shift studies in which performance was unimpaired by interchanging the features that were relevant and irrelevant after several trials of exposure (Bower \& Trabasso, 1963, 1964; Trabasso \& Bower, 1964, 1966).

Over the last two decades, an overwhelming amount of evidence supporting the view that people acquire well-defined concepts through a process of hypothesis testing has been amassed (see Levine, 1975). However, evidence that indicates that performance can be importantly affected by the frequency with which stimulus features occur has also been accumulating (Bourne, Ekstrand, Lovallo, Kellogg, Hiew, \& Yaroush, 1976; Johnson \& Toppino, 1974; Schultz \& Dodd, 1972). It has been suggested that people store information about feature frequency and use it to generate hypotheses (e.g., Kellogg, 1980). If so, an important change in the assumptions underlying hypothesis theory would be required. In addition, the possibility is raised that the processes associated with the use of feature frequency are common to the formation of both well-defined and illdefined concepts. For the present, however, it is important to understand better when and why feature frequency affects the acquisition of well-defined concepts. That is the purpose of this paper.

Haygood, Harbert, and Omlor (1970) provided one of the earliest indications that feature frequency might influence the acquisition of well-defined concepts. Using a feature-identification paradigm (conceptual rule known, relevant features unknown), they manipulated intradimensional variability (i.e., the number of features that can represent each dimension). As this variable was increased from two to six features per dimension, the frequency with which relevant features occurred remained constant while that of irrelevant features decreased. Their results indicated that conjunctive concepts were acquired more easily as a function of increasing intradimensional variability.

Haygood et al. (1970) proposed two explanations for their findings. First, subjects might store the frequency with which stimulus features occur and use this information to select the more frequently occurring features for testing. As the frequency of irrelevant features decreased, the frequency differential between relevant and irrelevant features would become greater. Thus, the high frequency of relevant features would be more discriminable, and they would be more likely to be selected for testing.

The second proposal by Haygood et al., (1970) was that subjects employed a hypothesis-testing strategy, in which they used logical information provided by positive instances to eliminate irrelevant features from consideration. In this case, decreasing the frequency of irrelevant features should improve performance because it increases the logical informational value of positive instances. That is, because irrelevant features can be disconfirmed logically whenever they fail to occur in a positive instance, a lower frequency of occurrence means that they will have a higher probability of being disconfirmed by any given positive instance and that they will be disconfirmed sooner and more often in a sequence of positive instances.

The frequency hypothesis of Haygood et al. (1970) received some support from a study by Chumbley, Lau, Rog, and Haile (1971). In one set of conditions, these investigators allowed the frequency differential favoring relevant features to increase in normal fashion with increasing intradimensional variability. In another set of conditions, one feature on each irrelevant dimension was held at a constant high frequency regardless of the level of intradimensional variability. The latter conditions impaired performance relative to the former, although increasing intradimensional variability improved performance in both cases.

In a similar but more analytical experiment, Schultz and Dodd (1972, Experiment 2) obtained somewhat different results that seemed to provide stronger support for the frequency hypothesis. In attempting to contrast the two hypotheses offered by Haygood et al. (1970), Schultz and Dodd found evidence that subjects did eliminate irrelevant features logically on the basis of positive-instance information. However, even when the number of successive positive instances required to eliminate all irrelevant features was held constant, feature frequency affected performance. Mean errors to criterion decreased as a function of increasing intradimensional variability as long as the latter variable produced the usual decrease in the frequency of irrelevant features. However (and in contrast to the findings of Chumbley et al., 1971), this effect was eliminated by having one feature of each irrelevant dimension occur with high frequency regardless of the level of intradimensional variability. Thus, Schultz and Dodd's data seemed to indicate that increasing intradimensional variability facilitates performance only when it leads to an increase in the size of the frequency differential favoring the relevant features.

Implicit in the frequency hypothesis of Haygood et al. (1970) was the assumption that subjects selected features to test on the basis of their frequency of occurrence in all stimuli, with no distinction being made between occurrence in positive and negative instances. Although Schultz and Dodd (1972) concluded (implicitly) in favor of this hypothesis, they had allowed frequency to covary in the positive and the negative categories. Thus, their findings could be attributed to frequency in the positive category alone. Indeed, Bourne et al. (1976) proposed a theory according to which subjects keep separate counts of the frequency with which features occur in the posi- 
tive and negative categories, respectively, and in which performance is determined primarily by the size of the frequency differential within the positive category. The original impetus for our research was to determine whether performance was affected by feature frequency in the negative category.

Despite the lack of systematic evidence, Schultz and Dodd's (1972) data did contain at least one indication that frequency in the negative category may have had important effects. In considering conditions with high intradimensional variability (six features per dimension), one condition had a large frequency differential in favor of the relevant features, whereas a second condition did not, due to the frequent occurrence of one feature on each irrelevant dimension. Because Schultz and Dodd attempted to control the informational value of positive instances, these conditions were virtually identical to one another with regard to feature frequency in the first few positive instances. In spite of this, when the overall frequency differential was large, subjects appeared to solve within the first several trials, whereas this was not true in the other condition. Thus, the frequency with which features occurred in the negative category may have produced the difference in performance between these conditions.

How might the frequency of irrelevant features in the negative category influence performance? One answer, of course, was suggested by Haygood et al. (1970) and by Schultz and Dodd (1972): Subjects might adopt a strategy in which they select hypotheses on the basis of the frequency with which features occur in all stimuli (positive and negative instances combined). However, there are several other possible explanations, all of which depend in part on a previously unexplored correlate of frequency. As the frequency of irrelevant features decreases, the similarity of stimuli occurring near one another in a stimulus sequence also decreases. That is, there is a decrease in the degree to which neighboring stimuli share common features. The similarity among neighboring positive instances is closely related to the rate at which positive instances allow irrelevant features to be eliminated logically. However, in the present context, we are concerned primarily with the potential effects of the similarity between negative instances and neighboring positive instances (intercategory similarity).

The first possible effect of intercategory similarity is that, under certain circumstances, subjects may be able to classify stimuli correctly without knowing the relevant features. For example, due to the attempt to control the informational value of positive instances in Schultz and Dodd's (1972) experiment, neighboring positive instances tended to be very similar to one another in all conditions, whereas the similarity between positive instances and neighboring negative instances decreased markedly with decreases in the frequency of irrelevant features. If subjects have any bias to classify similar stimuli into the same category and different stimuli into different categories, such a situation could affect results without necessarily affecting the true difficulty of isolating the relevant features. That is, subjects in lower frequency conditions would be expected to make fewer errors prior to solution attainment, and they would be more likely to begin their criterion run of successive correct responses before they actually knew the relevant features.

A second possible effect of intercategory similarity suggests how frequency in the negative category can affect actual solution attainment even though subjects may use a strategy such as that proposed by Bourne et al. (1976), in which the relevant features are identified primarily on the basis of a frequency discrimination within the positive category. According to Bourne et al., subjects adjust frequency counts for the positive or negative category after feedback is received on each trial. As it stands, their theory predicts little or no effect of frequency in the negative category or of intercategory similarity. However, there is some evidence that frequency counting is an automatic aspect of encoding (Hasher \& Zacks, 1979). If we supplement their theory with the assumption that classifying a stimulus at the time of its presentation involves encoding it as a positive or a negative instance, it is reasonable to expect inappropriate frequency adjustments to occur when subjects misclassify stimuli. Thus, assuming that intercategory similarity affects the probability of presolution classification errors (as we suggested in the last paragraph), frequency counts should become less accurate and solution attainment should decline as the frequency of irrelevant features and intercategory similarity increase.

The third possible effect of intercategory similarity involves memory for positive instances. There is evidence both for the importance of positive instances in conjunctive concept formation (e.g., Bourne \& Guy, 1968) and for the importance of memory for the information provided by previous instances (e.g., Bourne, Goldstein, \& Link, 1964). Any variable that would interfere with memory for positive-instance information could be expected to impair performance. Intercategory similarity may be such a variable. Under conditions of high intercategory similarity, negative instances may interfere with memory for positive instances or at least interfere with memory for whether features occurred in positive or in negative instances. As intercategory similarity decreases, interference would be expected to decrease, and performance should improve.

\section{EXPERIMENT 1}

To begin our investigation of the role of feature frequency in negative instances, different groups of subjects received a series of six-trial problems in 
which irrelevant features occurred either with low or high frequency. When frequency counts were based on the occurrence of features in all stimuli (both positive and negative), the low- and high-frequency problems were comparable to those used by Schultz and Dodd (1972) in conditions having large and small frequency differentials, respectively. However, frequency and logical informational value within the positive category were held strictly constant so that any differences between the groups would indicate that performance was affected by frequency within the negative category or by its correlate, intercategory similarity. In addition, although the subjects classified each stimulus as it was presented and received feedback, solution attainment was determined by whether the subjects could name the two relevant features after all six stimuli had been presented. This allowed us to assess the relationship between classification accuracy and solution attainment.

\section{Method}

Subjects and Design. The subjects were 54 undergraduate students who were paid for their participation in the experiment. The subjects were assigned randomly to one of two experimental groups (low or high frequency).

Stimulus materials. The stimulus dimensions and the dimensional features were identical to those employed by Haygood et al. (1970) and by Schultz and Dodd (1972). Each stimulus was a slide containing four letters and one digit arranged horizontally. Each of the five positions in which the letters and digit could appear represented a dimension, and each dimension could be represented by six possible values or features. The six features that could appear in each position (going from left to right) were the following: in the first position, the letters $A$ through $F$; in the second position, the letters $\mathrm{G}$ through $\mathrm{L}$; in the third position, the letters $M$ through $R$; in the fourth position, the letters $S$ through $\mathrm{X}$; and in the fifth position, the digits 1 through 6 .

Nine problems were constructed for each of the experimental conditions (high and low frequency). Each problem consisted of three positive and three negative instances. The three positive instances contained enough information for solution once all three stimuli had been viewed. One negative instance contained the first relevant feature but not the second, one contained the second relevant feature but not the first, and one contained neither relevant feature.

A new pair of relevant features was chosen randomly for each of the nine problems, with the restrictions that each dimension was relevant in at least three problems, no pair of dimensions was relevant in more than one problem, and no feature on any dimension was relevant in more than one problem. Each problem had a unique stimulus sequence representing one of the nine sequences of three positive and three negative instances that were possible when the following restrictions were in force: (1) all sequences had to end with a positive instance and (2) no more than two positive instances could occur in succession. The former restriction insured that the solution could not be determined logically before the final trial, when all stimuli had been viewed by the subject. The latter restriction was employed on grounds that a block of three successive positive instances might unduly bias subjects to use positiveinstance information only.

Each subject received only three of the nine problems; the three problems were selected such that each dimension was relevant at least once and such that not all three problems started with the same kind of stimulus (i.e., positive or negative instance). A counterbalancing procedure was used so that when the entire group of subjects was considered, each of the nine problems was presented as the first, second, or third problem equally often.
The frequency variable was manipulated in such a way that the nine problems employed in the high-frequency condition were exactly the same as the nine problems employed in the low-frequency condition, except for the negative instances. In all problems, one feature on each irrelevant dimension occurred in two positive instances. In the low-frequency condition, these irrelevant features did not occur in any negative instance. Thus, the most frequent irrelevant features occurred in .33 of a problem's six stimuli. In the high-frequency condition, the irrelevant features that occurred in two positive instances also occurred in two negative instances. Thus, the most frequent irrelevant features occurred on .66 of the stimuli in a problem, the same frequency of occurrence as the relevant features when frequency counts were based on all stimuli regardless of category.

Procedure. The subjects participated either individually or in groups of two or three. They received detailed instructions that explained the nature of the problem, the entire stimulus population, and the conjunctive rule. The stimulus population and the rule were summarized on cards that were continuously available to subjects throughout the experiment. To ensure knowledge of the rule, a pretraining task was used. Two relevant features were named, and then subjects had to classify six stimuli (three positive and three negative) in accordance with those features and the conjunctive rule. Then, two more relevant features were named, and the procedure was repeated. Following pretraining, the subjects received three successive six-trial feature-identification problems. Instructions emphasized that they should strive for accuracy both in classifying stimuli during a problem and in identifying the relevant features at the end of the problem. When each stimulus was presented, it remained on the screen until the subjects pressed a button labeled "yes" or a button labeled "no" to classify the stimulus as either a positive or a negative instance. As soon as the subjects responded, a feedback light was illuminated above the correct button. After $2.5 \mathrm{sec}$, the feedback light and the stimulus were terminated. Five seconds later, the next stimulus was presented. Following the subjects' responses to the final stimulus of a problem, a question mark appeared on the screen to signal the subjects that they should try to write down the relevant features. They were encouraged to guess if they were uncertain, but feedback regarding their accuracy was not provided until the experiment was over.

\section{Results and Discussion}

Initial analyses indicated that, compared with the subjects in the low-frequency/low-intercategorysimilarity condition, the subjects in the high-frequency/ high-intercategory-similarity condition made more classification errors $[t(52)=6.86, p<.001]$ and solved fewer problems, although the latter result was only marginally significant $[\mathrm{t}(52)=1.73, \mathrm{p}<.10]$. However, closer examination of the data suggested that the results depended upon whether problems started with a positive or a negative instance. Therefore, two analyses of variance were conducted using frequency/intercategory-similarity (high or low) as a between-subjects factor and type of start stimulus (positive or negative) as a within-subject factor. The dependent measure for one analysis was the percentage of trials involving a classification error (percent error trials), and, for the other, it was the percentage of problems in which the relevant features were correctly identified (percent problems solved).' Table 1 summarizes the data.

The important outcome of both analyses was a significant two-way interaction: $\mathrm{F}(1,52)=7.18$, $\mathrm{MSe}=$ .129 , for the percent problems solved, and $F(1,52)=$ 
Table 1

Mean Percentage of Problems Solved and Mean Percentage of Error Trials in Experiment 1 as a Function of Frequency/ Similarity (High or Low) and Type of Start Stimulus

\begin{tabular}{ccc}
\hline & Problems Solved & Error Trials \\
\hline Low & \multicolumn{3}{c}{ Positive Start } \\
High & 64.8 & 12.0 \\
& 68.5 & 42.0 \\
Low & \multicolumn{3}{c}{ Negative Start } \\
High & 68.5 & 29.9 \\
\hline
\end{tabular}

8.90, $\mathrm{MSe}=.026$, for the percent error trials (both ps < .025). However, Newman-Keuls comparisons among the four treatment conditions indicated that the nature of the interaction was different for the two dependent measures. The percent problems solved was lower for the high-negative (high-frequency/negativestart-stimulus) problems than for the high-positive, low-positive, and low-negative problems, which did not differ from one another. Thus, high frequency impaired solution attainment only when problems began with a negative instance. In terms of percent error trials, high-positive and high-negative problems did not differ from one another, but both produced more errors than did low-negative problems, which, in turn, produced more errors than did low-positive problems. These findings indicate that intercategory similarity affected the likelihood of making presolution errors, but that the number of presolution errors was not systematically related to solution attainment. In fact, a detailed examination of the data revealed no pattern of errors that was consistently related to whether or not the relevant features were identified correctly.

\section{EXPERIMENT 2}

In light of our findings in Experiment 1, it is interesting that all of Schultz and Dodd's (1972) problems apparently began with a negative instance. ${ }^{2}$ This makes the generality of their findings suspect. Yet any conclusions about Schultz and Dodd's findings based on our previous experiment would be somewhat questionable because of numerous procedural differences between our experiment and theirs.

In Experiment 2, we used procedures similar to those employed by Schultz and Dodd (1972). We partially replicated their experiment and extended it by varying the type of start stimulus.

\section{Method}

Subjects and Design. The subjects were 102 volunteers from introductory psychology classes. Six subjects were dismissed from the experiment for failure to solve within 70 trials. The remaining 96 subjects were assigned randomly in blocks of 8 to the eight experimental conditions. The latter represented the factorial combination of three bileveled independent variables: intradimen- sional variability (two or six features per dimension), type of start stimulus (positive or negative), and the frequency of irrelevant features (control or high frequency). In control-frequency conditions, the frequency of irrelevant features decreased in a normal fashion with increasing intradimensional variability. In highfrequency conditions, one feature on each irrelevant dimension occurred in .66 of the stimuli, regardless of intradimensional variability.

Stimulus materials. For conditions involving six features per dimension, the stimulus population was the same as for Experiment 1. For conditions involving two features per dimension, stimuli included only the first two features of each dimension (i.e., $A$ and $B, G$ and $H, M$ and $N, S$ and $T, 1$ and 2 ).

The problems were the same as those employed by Schultz and Dodd (1972, Experiment 2) in their "3-SST conditions," in which, beginning with any positive instance, three positive instances were required to logically disconfirm each irrelevant feature at least once. The relevant features for all problems were $G$ and $S$. In conditions starting with a negative instance, Schultz and Dodd's stimulus sequences were used without any modification. In conditions starting with a positive instance, Schultz and Dodd's (1972) sequences were modified by reversing the order of the initial two stimuli so that the first stimulus was positive and the second was negative. No other modification was made.

Procedure. The procedure was the same as that for Experiment 1 with regard to instructions, pretraining, and the nature and timing of stimulus presentation, subject responses, and feedback presentation. However, each subject received only one featureidentification problem, which could be up to 70 trials long. The subjects did not name the relevant features at the end of a problem. Rather, they continued to classify stimuli and receive feedback until they had attained a criterion of 16 consecutive correct responses or until they had received 70 trials without reaching criterion.

\section{Results and Discussion}

Since trials and errors to criterion produced the same results, only the error data are reported. See Figure 1.

As expected, we replicated Schultz and Dodd's (1972) findings for problems that started with a negative instance. That is, there was an intradimensional variability $x$ frequency interaction $[F(1,44)=4.72$, $\mathrm{MSe}=27.52]$. Analyses of simple main effects indicated that performance improved with increasing intradimensional variability for control-frequency conditions $[F(1,44)=4.75, p<.05]$, but not for highfrequency conditions $[F(1,44)<1.00]$. In contrast, when problems started with a positive instance, we did not replicate Schultz and Dodd's findings. Performance improved with increasing intradimensional variability for both frequency conditions. This was evidenced by a significant main effect of intradimensional variability $[\mathrm{F}(1,44)=4.20, \mathrm{p}<.05]$ and a nonsignificant interaction $[\mathrm{F}(1,44)<1.00](\mathrm{MSe}=17.86$, in both cases).

The different pattern of results obtained with positive and negative start stimuli were attributable primarily to the six-feature/high-frequency conditions, in which performance was much better when problems started with a positive rather than with a negative instance. [The difference was significant: $t(22)=$ $2.10, \mathrm{p}<.05]$. In fact, if one considers only conditions involving six features per dimension, the results 

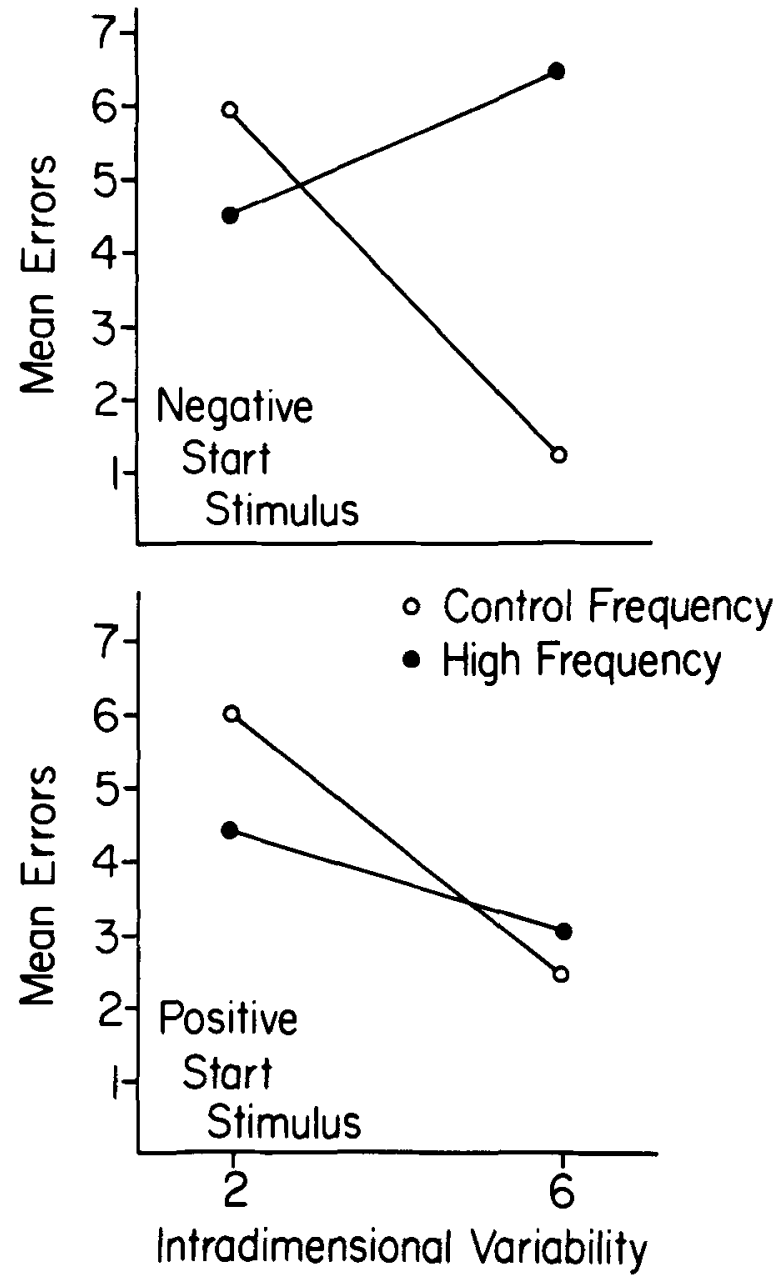

Figure 1. Mean errors to criterion in Experiment 2 as a function of intradimensional variability, frequency, and type of start stimuulus.

were remarkably similar to those we obtained in Experiment 1 . That is, the combination of high frequency and a negative start stimulus seemed to impair performance relative to the other conditions. It is unclear why a similar pattern of results failed to emerge in conditions involving two features per dimension, but that remains a problem for future research. At present, it is the conditions involving six features per dimension that are of primary interest because they are most comparable to the conditions employed in our previous experiment. The present results indicate that our previous findings were not limited to the somewhat unorthodox procedures that we employed.

Finally, the present results reflect on the adequacy of trials and errors to criterion as indicators of acquisition difficulty when conditions vary with respect to intercategory similarity. In conditions involving six features per dimension and control frequency, fully half $(.50)$ of the subjects (collapsed across start stimulus) made either no errors at all or only one error on the first trial. The comparable proportions of subjects for the other intradimensional variabilityfrequency conditions were $.08, .12$, and .04 for the six-feature/high-frequency, two-feature/controlfrequency, and two-feature/high-frequency conditions, respectively. The difference was reliable $\left[\chi^{2}(3)=21.06, p<.001\right]$. Since these "immediate solvers" could not have known the relevant features when they began their criterion runs, we interpret these data as evidence that low intercategory similarity can increase the probability of correctly classifying stimuli independently of whether or not the relevant features have been identified.

\section{GENERAL DISCUSSION}

We found that high frequency of irrelevant features in the negative category was associated with impaired acquisition of conjunctive concepts, but only when problems started with a negative instance. Although the type of start stimulus has received some attention in the concept literature (e.g., Giambra, 1971; Taplin, 1971), previous findings provided little a priori reason to expect the results that we obtained. Nevertheless, our findings have some noteworthy implications.

At the empirical level, the type of start stimulus may provide an explanation of why Chumbley et al. (1971) and Schultz and Dodd (1972) obtained different results in similar experiments. Recall that both studies varied intradimensional variability while holding the frequency of one feature on each irrelevant dimension at a high, constant level. Schultz and Dodd, who used the unusual procedure of starting each problem with a negative instance, found that holding the frequency of irrelevant features high eliminated the effect of intradimensional variability, whereas Chumbley et al. found that increasing intradimensional variability continued to be beneficial. It is interesting, therefore, that our second experiment replicated Schultz and Dodd's findings for problems that started with a negative instance but were more similar to the findings of Chumbley et al. for problems that started with a positive instance.

At the theoretical level, our findings are not reconciled easily with theories that people store featurefrequency counts and use this information to generate hypotheses. If one based frequency counts on the positive and negative categories combined, we should have found that high frequency impaired performance regardless of the type of start stimulus. If one based frequency counts on the positive category only, then, in Experiment 1, there should have been no effect of frequency because positive instances were identical for both conditions. One exception to the latter prediction might occur if subjects store incorrect frequency information every time they misclassify a stimulus. However, in Experiment 1, in 
which classification accuracy and solution attainment were determined separately, we were unable to find any pattern of classification errors that would account for the solution attainment results. In the final analysis, the major problem for frequency theories is that they have no mechanism that can account for the effect of type of start stimulus.

We believe that our findings can be explained by a hypothesis-testing theory, in which subjects acquire conjunctive concepts by selecting hypotheses on the basis of their memory for previous positive instances and by evaluating hypotheses on the basis of logical information. In this theoretical context, we propose that intercategory similarity, rather than frequency in the negative category, contributed to our results. Specifically, we hypothesize that a negative start stimulus proactively interferes with memory for the first positive instance of a problem, but that the interference is negligible unless negative instances are similar to positive instances (high intercategory similarity).

There are a number of reasons why performance could be importantly affected by whether or not subjects can remember the first positive instance accurately. First, if subjects can remember the features of the first positive instance, they might process information in a way that approximates the use of an ideal focusing or wholist strategy (Bruner, Goodnow, \& Austin, 1956). With this very efficient strategy, a subject's first hypothesis set consists of all the features of the first positive instance. Because this set must include the relevant features, solution attainment simply requires eliminating the irrelevant features from the hypothesis set. To the extent that subjects cannot remember the features of the first positive instance, they may be less likely to include the relevant features in their initial hypothesis set and may be more likely to resort to the use of a less efficient, successive-hypothesis-testing strategy.

Second, in selecting a new set of hypotheses, logical subjects who are not perfect focusers may scan their memory of previous trials in an attempt to select hypotheses that are consistent with previous information (Bruner et al., 1956). Thus, hypothesis selection should be more accurate to the extent that subjects can remember positive instances. In view of the poor memory that subjects exhibit for most stimuli (e.g., Bourne \& O'Banion, 1969), the first positive instance may be one of the few positive instances with a chance to be remembered well enough to affect performance.

The theory that we favor stresses the importance of logical hypothesis testing as opposed to the storage and use of frequency information. Yet, the frequency with which irrelevant features occur in positive instances is an important variable according to both logical and frequency theories. It is informative, therefore, to compare these viewpoints with respect to their theoretical differences and the resulting implications.

One major theoretical difference concerns the consequences of a feature's failure to occur in positive instances. Feature frequency and the size of the frequency differentials among features vary along a continuum. That is, in conjunctive problems, the likelihood of selecting a feature should decrease continuously with the number of times it has failed to occur in a positive instance. In contrast, the use of logic suggests that features can be in only one of two discrete states of consideration. They can be tenable if they have appeared in all positive instances. Or, they can be rejected if they have failed to occur in one positive instance, but they are equally rejected regardless of the number of positive instances in which they have failed to appear.

The all-or-none characteristic of logical processing, however, describes logical competence that is limited in practice by performance factors, particularly by memory. Without perfect memory for previous stimuli and hypotheses, the probability that a feature will be considered tenable or rejected may vary continuously with its frequency of occurrence. That is, the less often a feature occurs in positive instances, the more likely subjects will be to remember that the feature was rejected on at least one trial. Thus, like frequency theory, a view emphasizing logical processing would predict that the probability that features are selected for testing would vary continuously with their frequency of occurrence.

Another theoretical difference concerns the nature of the information stored in memory that is used to select hypotheses. An emphasis on logical processing has generally been associated with the use of memory for previous stimuli and for previously tested hypotheses. Frequency theories, on the other hand, usually imply that subjects use a more abstract form of frequency information which is an independent attribute of memory. It preserves feature frequency without preserving information that differentiates specific occurrences of a feature in different stimuli. This theoretical difference yields different predictions. The logical-processing view, with its emphasis on memory for stimulus information, predicts that performance should be affected by factors that influence the memorability of stimulus information even though they do not influence feature-frequency counts-a prediction that is consistent with our findings. No such effect is predicted by frequency theory. However, whether these predictions derive from an irreconcilable theoretical difference is uncertain because frequency theorists have not committed themselves to a precise description of how frequency information is represented (Bourne et al., 1976, p. 311). The different predictions may not reflect a substan- 
tive theoretical disagreement as much as they reflect the current state of development of frequency theory.

Finally, a methodological comment is in order. Trials and errors to a criterion of successive correct classifications have been the most common dependent measures employed in feature-identification studies. These measures have been interpreted as indicators of how long subjects take to identify the relevant features (i.e., as indicators of acquisition difficulty). However, the results of Experiment 1 showed that intercategory similarity can affect classification errors without necessarily affecting solution difficulty, and Experiment 2 indicated that intercategory similarity can affect the probability of beginning the criterion run before the relevant features have been identified. These findings suggest that investigators should be cautious in interpreting results based on trials or errors to criterion. These measures may not reflect accurately the differences in acquisition difficulty among conditions unless the conditions are comparable with regard to the similarity relations that exist among stimuli both within and between categories.

\section{REFERENCES}

Bourne, L. E., Jr., Exstrand, B. R., Lovallo, W. R. Kelloga, R. T., Hiew, C. C., \& Yaroush, R. A. Frequency analysis of attribute identification. Journal of Experimental Psychology: General, 1976, 105, 294-312.

Bourne, L. E., Jr., Goldstein, S., \& Link, W. E. Concept learning as a function of availability of previously presented information. Journal of Experimental Psychology, 1964, 67, 439-448.

Bourne, L. E., Jr., \& Guy, D. E. Learning conceptual rules: II. The role of positive and negative instances. Journal of Experimental Psychology, 1968, 67, 439-448.

Bourne, L. E., Jh., \& O'BAnion, K. Memory for individual events in concept identification. Psychonomic Science, 1969, 16, 101-103.

Bower, G. H., \& Trabasso, T. Reversals prior to solution in concept identification. Journal of Experimental Psychology, $1963,66,409-418$.

Bower, G. H., \& Trabasso, T. Concept identification. In R. C. Atkinson (Ed.), Studies in mathematical psychology. Stanford: Stanford University Press, 1964.

Bruner, J. S., Goodnow, J. J., \& Austin, G. A. A study of thinking. New York: Wiley, 1956.

Chumbley, J., Lau, P., Rog, D., \& Haile, G. Concept identification as a function of intradimensional variability, availability of previously presented material, and relative frequency of relevant attributes. Journal of Experimental Psychology, 1971, 90, 163-165.

Chumbley, J. I., Sala, L. S., Bourne, L. E., Jr. Bases of acceptability ratings in quasinaturalistic concept tasks. Memory \& Cognition, 1978, 6, 217-226.

Ginmbra, L. M. Selection strategies for eight concept rules with exemplar and nonexemplar start cards: A within-subjects replication. Journal of Experimental Psychology, 1971, 87, 143-145.

Goldman, D., \& Homa, D. Integrative and metric properties of abstracted information as a function of category discriminability, instance variability, and experience. Journal of Experimental Psychology: Human Learning and Memory, 1977, 3, 375-385.

HAsHER, L., \& ZACKs, R. T. Automatic and effortful processes in memory. Journal of Experimental Psychology: General, 1979, 108, 356-388.

Hayes-Roth, B., \& Hayes-Roth, F. Concept learning and the recognition and classification of exemplars. Journal of Verbal Learning and Verbal Behavior, 1977, 16, 321-338.

Haygood, R. C., Harbent, T. L., \& Omlor, J. A. Intradimensional variability and concept identification. Journal of Experimental Psychology, 1970, 83, 216-219.

Hull, C. L. Quantitative aspects of the evaluation of concepts: An experimental study. Psychological Monographs, 1920, 28 (1, Whole No. 123).

Johnson, P. J., \& Toppino, T. C. Effects of category attention, relative frequency of relevant values, and practice on attribute identification performance. Journal of Experimental Psychology, 1974, 103, 160-166.

Kellogg, R. T. Feature frequency and hypothesis testing in acquisition of rule-governed concepts. Memory \& Cognition, 1980, 8, 297-303.

Kellogg, R. T., Bourne, L. E., JR., \& Ekstrand, B. R. Feature frequency and the acquisition of natural concepts. American Journal of Psychology, 1978, 91, 211-222.

Levine, M. A cognitive theory of learning. New York: Erlbaum, 1975.

Neumann, P. G. An attribute frequency model for the abstraction of prototypes. Memory \& Cognition, 1974, 2, 241-248.

Restle, F. A. A theory of discrimination learning. Psychological Review, 1955, 62, 11-19.

Restle, F. A. The selection of strategies in cue learning. Psychological Review, 1962, 69, 329-343.

Schultz, R. G., JR., \& DoDD, D. H. Intradimensional variability in concept identification: A replication, extension, and partial clarification of the Haygood, Harbert, and Omlor findings. Journal of Experimental Psychology, 1972, 94, 321-325.

TAPLIN, J. E. Effect of initial instance on attribute identification of concepts using a selection procedure. Journal of Experimental Psychology, 1971, 88, 177-181.

Trabasso, T., \& Bower, G. H. Presolution reversal and dimensional shifts in concept identification. Journal of Experimental Psychology, 1964, 67, 398-399.

Trabasso, T., \& Bower, G. H. Presolution dimensional shifts in concept identification: A test of the sampling with replacement axiom in all-or-none models. Journal of Mathematical Psychology, 1966, 3, 163-173.

Trabasso, T., \& Bower, G. H. Attention in learning. New York: Wiley, 1968.

\section{NOTES}

1. Percentages were used because some subjects had two problems starting with a positive instance and one starting with a negative instance, whereas other subjects had the opposite arrangement. To allay concerns that this dependent measure would produce serious violations of the assumptions underlying the analysis of variance, the main results of the reported parametric analyses were confirmed by the results of appropriate nonparametric analyses.

2. We thank Roger Schultz and David Dodd for making their stimulus sequences available to us.

(Manuscript received January 22, 1982; revision accepted for publication March 28, 1983.) 